\title{
ESCARABAJOS FITÓFAGOS (COLEOPTERA: SCARABAEIDAE) DEL DEPARTAMENTO DEL ATLÁNTICO, COLOMBIA
}

\author{
SANDY GARCÍA-ATENCIA ${ }^{1}$ y NEIS MARTÍNEZ-HERNÁNDEZ ${ }^{2}$
}

\author{
${ }^{1}$ Grupo de investigación Insectos de Colombia, Instituto de Ciencias Naturales, Universidad Nacional de \\ Colombia. Av. carrera 45 No 26-85, Bogotá D.C., Colombia. <sandyga.01@gmail.com> \\ ${ }^{2}$ Grupo de Investigación Biodiversidad del Caribe colombiano. Departamento de Biología, Facultad de Ciencias \\ Básicas, Universidad del Atlántico, km 7 antigua vía a Puerto Colombia. Estudiante Doctorado en Ciencias- \\ Biología. Departamento de Biología. Universidad Nacional de Colombia- Sede Bogotá. Correo electrónico: \\ <neyjosemartinez@gmail.com>; <sandyga.01@gmail.com> \\ Recibido: 05/08/2014; aceptado: 21/10/2014
}

García-Atencia, S. \& Martínez-Hernández, N. 2015. Escarabajos fitófagos (Coleoptera: Scarabaeidae) del Departamento del Atlántico, Colombia. Acta Zoológica Mexicana (n. s.), 31(1): 89-96.

RESUMEN. Se realizó un inventario de las especies fitófagas de la familia Scarabaeidae para el departamento del Atlántico, con el fin de actualizar la distribución de estos insectos en uno de los departamentos menos estudiados del país. Se realizaron recolectas en cinco fragmentos (Reserva Campesina La Montaña, RCM; Reserva Bijibana, RB; Corrales de San Luis, CSL; Distrito de Manejo Integrado Luriza, DMI Luriza; Campus Universidad del Atlántico, CUA) con ayuda de trampas de luz, carpotrampas y recolecta manual. Se obtuvieron 32 especies distribuidas en 18 géneros y cuatro subfamilias. Las subfamilias Rutelinae y Dynastinae son las más diversas con 11 especies cada una, seguidas por Melolonthinae con seis y Cetoniinae con cuatro. Se obtiene un dato puntual en la distribución de Xenopelidnota anomala en el país y se registra por primera vez Tomarus cuniculus. Lo anterior representa un aumento de riqueza genérica y específica para el departamento del Atlántico, lo que hace necesario incluir dentro de los proyectos ambientales el aumento de reservas naturales y socialización para el cuidado de la Biodiversidad, específicamente de escarabajos fitófagos de la familia Scarabaeidae que desempeñan roles ecológicos importantes dentro de los ecosistemas.

Palabras Clave: Scarabaeidae "Pleurosticti", Inventario, Distribución, Atlántico

\section{INTRODUCCIÓN}

Los escarabajos fitófagos de la familia Scarabaeidae son un grupo ampliamente diversificado en Colombia, pues se conocen 109 géneros y 595 especies (Restrepo et al. 2003; Gasca \& Amat 2010). Se caracterizan por ser de gran importancia económica y ecológica, debido a que desempeñan funciones importantes dentro de las comunidades bióticas por su papel en la polinización, la degradación y reciclaje de la materia orgánica (Morón 1985). Además, por su variedad de gremios tróficos pueden reflejar la dinámica de los ecosistemas, como es el caso de las especies sapro-xilófagas que cumplen un papel relevante en los bosques como degradadores primarios de madera (Morón 1994). En el caso de los Dynastinae Oryctini, las
García-Atencia, S. \& Martínez-Hernández, N. 2015. Phytophagous scarab beetles (Coleoptera: Scarabaeidae) from Department of Atlantico, Colombia. Acta Zoológica Mexicana (n. s.), 31(1): 89-96.

ABSTRACT. A phytophagous species inventory belonging to the family Scarabaeidae to the department of Atlantico was performed in order to update the distribution of these insects in one of the least studied departments of Colombia. Five fragments were sampled (Reserva Campesina La Montaña, RCM; Reserva Bijibana, RB; Corrales de San Luis, CSL; Distrito de Manejo Integrado Luriza, DMI Luriza; Campus Universidad del Atlántico, CUA) using light traps, traps baited with fruits and manually capture. Thirty-two species distributed in 18 genera and four subfamilies were collected. The subfamilies Rutelinae and Dynastinae were the most diverse with 11 species each one, followed by Melolonthinae with six and Cetoniinae with four. A data about the distribution of Xenopelidnota anomala in the country was obtained, and Tomarus cuniculus was registered for the first time. This represents an increasing of the generic and specific richness for the department of Atlantico, making it necessary to include environmental projects for increasing natural reservoirs, and socialization for Biodiversity care, specifically phytophagous scarab beetles belonging to the Scarabaeidae family that play important ecological roles within ecosystems.

Key words: Scarabaeidae "Pleurosticti”, Inventory, Distribution, Atlantico.

larvas son capaces de fragmentar grandes cantidades de madera durante su alimentación, acelerando el proceso de descomposición (Morón 1985). En el proceso, se producen detritos y excreciones que facilitan la acción de otros agentes descomponedores en la microfauna y microflora (Gasca \& Amat 2010).

Por otro lado, las larvas conforman complejos edafícolas cuyos hábitos alimenticios varían desde la rizofagia hasta la saprofagia (Pardo-Locarno et al. 2007). En su mayoría, son consideradas las causantes de daños importantes en cultivos vegetales por lo que en los últimos tiempos, han despertado el interés de las autoridades fitosanitarias en especial los géneros Astaena, Phyllophaga, Isonychus, Macrodactylus, Plectris, Barybas, Charioder- 
ma, Manopus y Ceraspis de la subfamilia Melolonthinae (Pardo-Locarno 2003c).

Con respecto a los estudios de estos escarabajos en Colombia, en los últimos años se han realizado informes sobre distribución (Suárez \& Amat 2007; Gasca \& Amat 2010), sinópsis taxonómicas y descripción de nuevas especies (Sanabria et al. 2012; Vallejo \& Wolff 2013, López-García et al. 2014). Por otro lado, la mayoría de trabajos ecológicos se centran en analizar la composición de especies en agro-ecosistemas (Útima \& Vallejo 2008), caracterizar los complejos regionales de plagas (Pardo Locarno 2003a, 2003b, 2003c; Pardo-Locarno et al. 2012) y describir estados inmaduros de algunas especies (Pardo-Locarno et al. 2007).

En las tierras bajas del Caribe colombiano, el conocimiento de los fitófagos de la familia Scarabaeidae es escaso y no existe un inventario reciente de especies. Existen reportes de distribución para el Atlántico, realizados por Restrepo et al. (2003), Suárez \& Amat (2010), Gasca \& Amat (2010); aunque se presume que faltan especies por incorporar en tales inventarios, debido a la falta de estudios con técnicas más específicas para este grupo o la poca intensidad en los muestreos. Ante la problemática anteriormente mencionada, el objetivo general de este trabajo fue realizar un inventario organizado de los escarabajos fitófagos del departamento del Atlántico; que permitiera no solo conocer las especies presentes, sino también su distribución. De esta manera se logró actualizar la información de estos insectos en uno de los departamentos menos estudiados de Colombia.

\section{MATERIALES Y MÉTODOS}

Área de estudio. En los fragmentos de Bosque seco tropical del departamento del Atlántico muestreados (Fig. 1), la vegetación predominante es la típica de Bosque seco, descrita por el IAvH (1998). Alrededor de los fragmentos son comunes las prácticas de ganadería extensiva y zonas de policultivo de Zea mays L. (Maíz), Manihot esculenta Crantz (Yuca), Capsicum annuum L. (Ají), Citrullus lanatus (Thunb.) Matsum. \& Nakai (Sandía) y actividades de quema de árboles para la obtención de carbón vegetal y extracción de leña, lo que ha reducido su área original. Además, presentan una precipitación media anual de $1051.7 \mathrm{~mm}$ y temperatura entre 27 y $28^{\circ} \mathrm{C}$.

Muestreos. La recolecta del material se realizó entre diciembre de 2012 y agosto de 2013 en cinco fragmentos de Bosque seco Tropical del departamento del Atlántico (Cuadro 1). Se recolectaron los adultos fitófagos de Scarabaeidae con ayuda de trampas de luz blanca y ultravioleta, que se colocaron en cada sitio alternadamente y operaban desde las 18:00 hasta las 6:00. Adicionalmente, se realizó

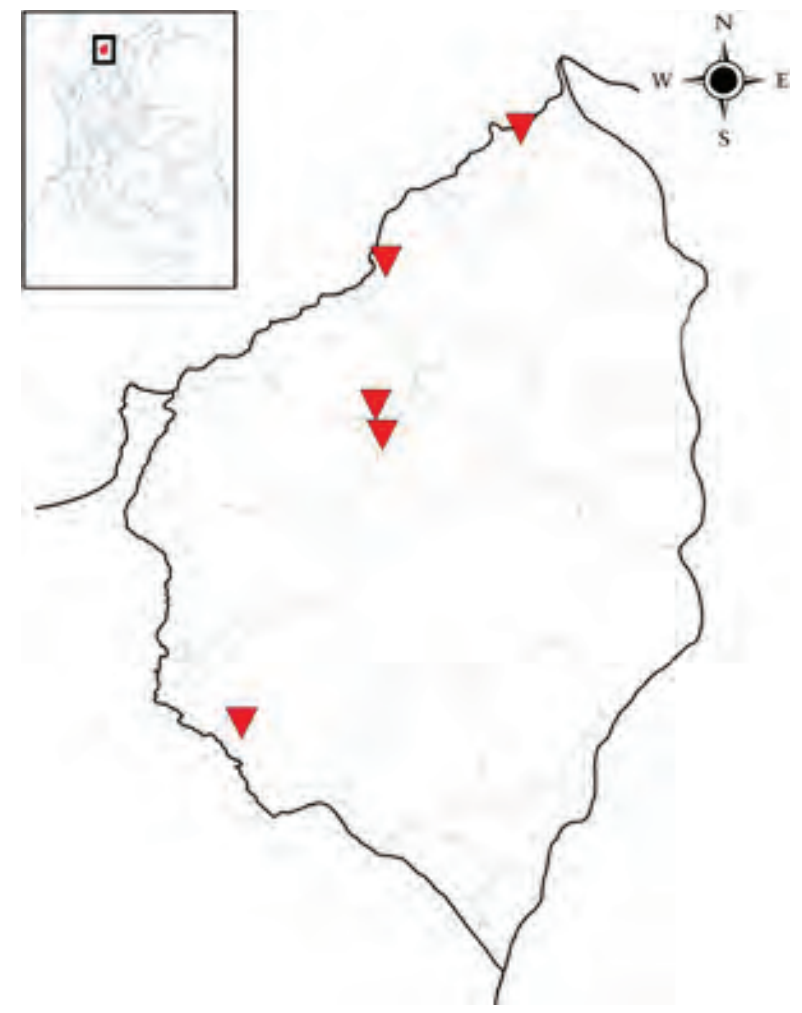

Figura 1. Ubicación de los Fragmentos de Bosque seco Tropical, muestreados en el departamento del Atlántico.

una revisión manual en sustratos para una inversión de 1hora/hombre. Los días de recolecta fueron seleccionados según el calendario lunar de forma que el muestreo nocturno se realizó con la máxima oscuridad.

Preservación e identificación de las muestras. Las muestras se preservaron en alcohol al 70\%. Los individuos se identificaron con las claves propuestas por Endrödi (1985), Ramírez-Ponce \& Morón (2009), Sanabria et al. (2012), Vallejo \& Wolff (2013). El material producto de este trabajo reposa en la Colección de la Universidad del Atlántico y en la Colección entomológica del Instituto de Ciencias Naturales (ICN-MHN)

\section{RESULTADOS}

Se recolectaron 32 especies, distribuidas en 18 géneros de cuatro subfamilias de Scarabaeidae. Dynastinae estuvo representada por seis géneros, Rutelinae y Melolonthinae por cuatro y Cetoniinae, por tres (Cuadro 2). Se incorporan doce géneros no reportados para el departamento del Atlántico (Cuadro 3), una nueva especie registrada para el país que corresponde a Tomarus cuniculus y una distribución puntual para Xenopelidnota anomala.

A continuación se indica la composición específica y características de las recolectas realizadas en la presente investigación (Ver figuras en el Apéndice) 
Cuadro 1. Fragmentos de estudio. Abreviaturas: RCM, Reserva Campesina La Montaña; CSL, Corrales de San Luis; DMI Luriza, Distrito de Manejo Integrado Luriza; RB, Reserva Bijibana; CUA, Campus Universidad del Atlántico.

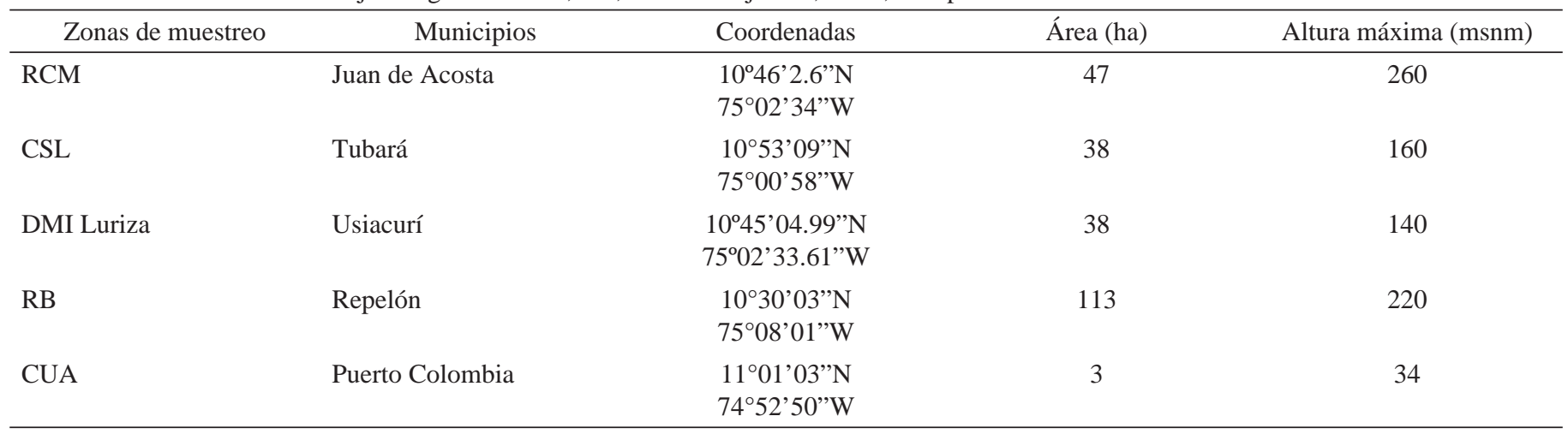

\section{CETONIINAE}

Gymnetini: Los representantes de la tribu se caracterizan por presentar la cabeza sin ornamentos o con proyecciones, carinas y fosetas más o menos discretas; lóbulo basal medio del pronoto redondeado, muy proyectado hacia atrás cubriendo el escutelo; apófisis preproesternal corta, delgada o ausente (Morón et al. 1997).

Cotinis barthelemyi (Gory \& Pecheron, 1833): Se recolectó con carpotrampa al interior del Bosque de la RB. Se encuentra en los departamentos del Atlántico, Magdalena y Caldas (Suárez \& Amat 2007).

Cotinis lebasi (Gory \& Pecheron, 1833): Se recolectó con carpotrampa al interior del Bosque de la RB. Es de amplia distribución en el país y para la Costa norte colombiana está citada para los departamentos de Cesar y Magdalena (Suárez \& Amat 2007)

Hoplopyga liturata (Olivier, 1789): Se recolectaron dos individuos (un macho y una hembra) con carpotrampas de frutas durante septiembre en el fragmento DMI Luriza. Es de amplia distribución en el país y para el Caribe colombiano sólo estaba reportada en Sucre (Suárez \& Amat 2007).

Cetoniini: Presentan cabeza sin ornamentos, cuernos o carinas; escutelo visible no cubierto por una expansión basal del pronoto; superficie dorsal variable, lisa, pulida, brillante, tomentosa. Preproesternón usualmente tectiforme, proyección mesoesternal normalmente ausente o al menos muy pequeña (Morón et al. 1997).

Euphoria yucateca Bates, 1889: Se recolectaron dos individuos macho con carpotrampa al interior del Bosque de la RB y RCM durante el mes de septiembre. Está ampliamente distribuida en el Caribe colombiano y Norte de Santander (Orozco 2012).

DYNASTINAE

Cyclocephalini: Forma del clípeo altamente variable. Carecen de tubérculos, depresiones, carinas o cuernos sobre la cabeza y el tórax; mandíbulas usualmente estrechas con el borde lateral curvado y sin dentículos; el mentón es más o menos elongado-ovalado no cubriendo la base de los palpos (Endrödi 1966).

Cyclocephala melanocephala (Fabricius, 1775): Se capturaron 15 individuos en los fragmentos RCM, DMI Luriza, RB y CUA con trampas de luz blanca y ultravioleta durante febrero, mayo, junio, julio y agosto. Es una especie de amplia distribución en Colombia.

Cyclocephala ovulum Bates, 1888: Se recolectaron 89 individuos presentes sólo en el fragmento RCM, capturados con trampas de luz blanca y ultravioleta. Su distribución en el país es poco conocida

Cyclocephala sp.: Se recolectó un individuo hembra en la RCM con trampa de luz blanca.

Dyscinetus dubius (Olivier, 1789): Se recolectaron dos individuos en la RCM con trampa de luz blanca. Se ha encontrado en zonas secas del Caribe colombiano tales como Cesar y Sucre (Pardo-Locarno et al. 2012)

Oryctini: Cuerpo grande y robusto, mandíbulas usualmente anchas y muy expuestas, con o sin dentículos en el borde externo, mentón ovalado y no cubre la base de los palpos labiales; propigido generalmente con estructuras estriduladoras; protibias con tres o cuatro dentículos grandes en su borde exterior; ápices de las metatibias normalmente con proyecciones agudas o dentículos grandes y sólo en algunos casos el margen es casi recto o truncado (Sanabria et al. 2012).

Strategus aloeus (Linnaeus, 1758): Se recolectaron cuatro individuos durante mayo en la RCM y CUA con trampas de luz blanca y ultravioleta. La especie es de amplia distribución en Colombia, considerada de importancia agrícola afectando cultivos de palmas (Araceae) (Sanabria et al. 2012).

Strategus jugurtha Burmeister, 1847: Se recolectó un individuos macho en la RB, con trampas de luz blanca en mayo. Su distribución abarca las cordilleras central y oriental de Colombia, Vichada y Magdalena (Sanabria et al. 2012). 
Cuadro 2. Listado de especies recolectadas en cinco fragmentos del departamento del Atlántico. RCM, Reserva Campesina La Montaña; CSL, Corrales de San Luis; DMI Luriza, Distrito de Manejo Integrado Luriza; RB, Reserva Bijibana y CUA, Campus Universidad del Atlántico.

*Nuevos reportes para el departamento del Atlántico; **Nuevos reportes para Colombia.

\begin{tabular}{|c|c|c|c|c|c|}
\hline \multirow[t]{2}{*}{ Especies } & \multicolumn{5}{|c|}{ Fragmentos } \\
\hline & RCM & CSL & DMI Luriza & R.B & CUA \\
\hline \multicolumn{6}{|l|}{ CETONIINAE S $=4$} \\
\hline \multicolumn{6}{|l|}{ Gymnetini } \\
\hline Cotinis barthelemyi (Gory \& Pecheron, 1833) & 0 & 0 & 0 & 1 & 0 \\
\hline Cotinis lebasi (Gory \& Pecheron, 1833)* & 0 & 0 & 0 & 1 & 0 \\
\hline Hoplopyga liturata (Olivier, 1789)* & 0 & 0 & 1 & 0 & 0 \\
\hline \multicolumn{6}{|l|}{ Cetoniini } \\
\hline Euphoria yисаteca Bates, 1889 & 1 & 0 & 0 & 1 & 0 \\
\hline \multicolumn{6}{|l|}{ DYNASTINAE $S=11$} \\
\hline \multicolumn{6}{|l|}{ Cyclocephalini } \\
\hline Cyclocephala melanocephala (Fabricius, 1775) & 1 & 0 & 1 & 1 & 1 \\
\hline Cyclocephala ovulum Bates, 1888* & 1 & 0 & 0 & 0 & 0 \\
\hline Cyclocephala sp & 1 & 0 & 0 & 0 & 0 \\
\hline Dyscinetus dubius (Olivier, 1789)* & 1 & 0 & 0 & 0 & 0 \\
\hline \multicolumn{6}{|l|}{ Oryctini } \\
\hline Strategus aloeus (Linnaeus, 1758)* & 1 & 0 & 0 & 0 & 1 \\
\hline Strategus jugurtha Burmeister, 1847* & 0 & 0 & 0 & 1 & 0 \\
\hline \multicolumn{6}{|l|}{ Pentodontini } \\
\hline Euetheola humillis (Burmeister, 1847)* & 1 & 0 & 0 & 1 & 0 \\
\hline Tomarus fossor (Latreille, 1833) & 1 & 0 & 0 & 1 & 1 \\
\hline Tomarus cuniculus (Fabricius, 1801)** & 0 & 0 & 0 & 1 & 0 \\
\hline \multicolumn{6}{|l|}{ Phileurini } \\
\hline Hemiphileurus sp & 0 & 1 & 0 & 0 & 1 \\
\hline Phileurus dydimus Linnaeus $1758 *$ & 1 & 0 & 0 & 0 & 0 \\
\hline \multicolumn{6}{|l|}{ MELOLONTHINAE S = 6} \\
\hline \multicolumn{6}{|l|}{ Melolonthini } \\
\hline Phyllophaga (Phyllophaga) menetriesi (Blanchard, 1851)* & 1 & 0 & 1 & 0 & 0 \\
\hline Phyllophaga impressipyga (Frey, 1975)* & 1 & 1 & 0 & 1 & 1 \\
\hline Phyllophaga pachypyga (Burmeister, 1855)* & 1 & 1 & 0 & 1 & 1 \\
\hline \multicolumn{6}{|l|}{ Macrodactylini } \\
\hline Liogenys quadridens (Fabricius, 1798)* & 1 & 1 & 1 & 0 & 0 \\
\hline Barybas sp & 1 & 1 & 0 & 1 & 0 \\
\hline \multicolumn{6}{|l|}{ Sericini } \\
\hline Astaena sp & 1 & 0 & 0 & 1 & 0 \\
\hline \multicolumn{6}{|l|}{ RUTELINAE S = 11} \\
\hline \multicolumn{6}{|l|}{ Anomalini } \\
\hline Paranomala inconstans (Burmeister, 1844)* & 1 & 0 & 1 & 0 & 0 \\
\hline Paranomala sp1 & 1 & 1 & 1 & 1 & 1 \\
\hline Paranomala sp2 & 1 & 0 & 1 & 1 & 0 \\
\hline Paranomala sp3 & 1 & 1 & 0 & 0 & 0 \\
\hline Paranomala sp4 & 1 & 0 & 1 & 0 & 0 \\
\hline \multicolumn{6}{|l|}{ Geniatini } \\
\hline Leucothyreus sp1 & 1 & 1 & 0 & 0 & 1 \\
\hline Leucothyreus sp2 & 1 & 1 & 0 & 1 & 0 \\
\hline Leucothyreus sp3 & 1 & 0 & 0 & 1 & 1 \\
\hline Leucothyreus sp4 & 1 & 0 & 0 & 0 & 0 \\
\hline \multicolumn{6}{|l|}{ Rutelini } \\
\hline Pelidnota polita Latreille, $1811^{*}$ & 1 & 1 & 0 & 0 & 0 \\
\hline Xenopelidnota anomala (Burmeister, 1844)* & 1 & 1 & 0 & 1 & 1 \\
\hline Total & 26 & 11 & 8 & 17 & 10 \\
\hline
\end{tabular}


Cuadro 3. Riqueza genérica de Scarabaeidae fitófagos en el departamento del Atlántico, Colombia, según Restrepo-Giraldo (2003), Suárez y Amat (2007), Gasca y Amat (2010) y presente estudio (2014). *Nuevos reportes para el departamento del Atlántico.

\begin{tabular}{|c|c|c|c|c|c|c|}
\hline Subfamilia & Tribu & Género & 2003 & 2007 & 2010 & 2014 \\
\hline \multirow[t]{2}{*}{ Cetoniinae } & Gymnetini & Cotinis & & $\mathrm{x}$ & & $\mathrm{x}$ \\
\hline & & Hoplophyga* & & & & $\mathrm{x}$ \\
\hline \multirow[t]{7}{*}{ Dynastinae } & Cyclocephalini & Ancognatha & $\mathrm{x}$ & & $\mathrm{x}$ & \\
\hline & & Cyclocephala & $\mathrm{x}$ & & & $\mathrm{x}$ \\
\hline & Oryctini & Strategus* & & & & $\mathrm{x}$ \\
\hline & Pentodontini & Euetheola* & & & & $\mathrm{x}$ \\
\hline & & Tomarus & $\mathrm{x}$ & & $\mathrm{x}$ & $\mathrm{x}$ \\
\hline & Phileurini & Hemiphileurus* & & & & $\mathrm{x}$ \\
\hline & & Phileurus* & & & & $\mathrm{x}$ \\
\hline & Macrodactylini & Barybas & & & & $\mathrm{x}$ \\
\hline & & Liogenys* & & & & $\mathrm{x}$ \\
\hline \multirow[t]{6}{*}{ Rutelinae } & Anomalini & Callistethus & $\mathrm{x}$ & & & \\
\hline & & Paranomala & $\mathrm{x}$ & & & $\mathrm{x}$ \\
\hline & Geniatini & Bolax & $\mathrm{x}$ & & & \\
\hline & & Leucothyreus* & & & & $\mathrm{x}$ \\
\hline & Rutelini & Pelidnota & $\mathrm{x}$ & & & $\mathrm{x}$ \\
\hline & & Xenopelidnota* & & & & $\mathrm{x}$ \\
\hline
\end{tabular}

Pentodontini: Presentan carinas, fosetas o tubérculos de dimensiones moderadas en la cabeza y/o pronoto. Mandíbulas usualmente anchas con o sin dentículos en el borde externo. Mentón alargado ovalado, que no cubre la base de los palpos labiales. Generalmente con tres procesos dentiformes en el borde externo de las protibias (Morón et al. 1997).

Euetheola humilis (Burmeister, 1847): Se recolectaron tres individuos en los fragmentos de RCM y RB, con trampas de luz ultravioleta. Su distribución es poco conocida.

Tomarus cuniculus (Fabricius, 1801): Recolectado al interior del bosque de la RB con trampa de luz blanca. Esta especie se registra por primera ocasión para Colombia. Su distribución abarcaba Norte américa, Centro américa, Venezuela y Brasil. El individuo revisado corresponde a una hembra.

Tomarus fossor (Latreille, 1833): Se recolectaron doce individuos en los fragmentos RCM, RB y CUA, con trampas de luz blanca y ultravioleta. Su distribución es Colombia es poco conocida, aunque es citada para el departamento del Atlántico (Restrepo et al. 2003).
Phileurini: Cuerpo robusto generalmente deprimido, con el protórax ampliamente separado del pterotórax; clípeo puntiagudo; mandíbulas estrechas con el borde externo dentado o sólo recurvado, y con el ápice y el borde interno provisto de dientes largos y agudos (Morón et al. 1997).

Hemiphileurus sp.: Se recolectaron tres individuos hembras con trampas de luz blanca y ultravioleta en CSL y CUA.

Phileurus didymus Linnaeus 1758: Se recolectaron dos individuos hembra y macho durante mayo en la RCM con trampas de luz blanca. Su distribución es poco conocida en Colombia, y para la Costa norte del país no presenta información. Para la costa caribe, se ha reportado en Córdoba (Pardo-Locarno et al. 2012).

MELOLONTHINAE

Melolonthini: Los adultos presentan el labro esclerosado, con el borde anterior grueso y normalmente bilobulado o reniforme, pero en vista dorsal queda cubierto por el borde del clípeo; mandíbulas bien desarrolladas, con los bordes exteriores engrosados; antenas con ocho a diez artejos (Morón et al. 1997). 
Phyllophaga impressipyga (Frey, 1975): Se recolectaron 403 individuos con trampas de luz blanca y ultravioleta. Estuvo presente en casi todos los fragmentos, excepto en DMI Luriza, Sólo se conocía su distribución para departamentos de Caldas, Cundinamarca y Valle del Cauca (Pardo-Locarno 2003, Vallejo \& Wolff 2013)

Phyllophaga (Phyllophaga) menetriesi (Blanchard, 1851): Se recolectaron 203 individuos en los fragmentos RCM y DMI Luriza, con trampas de luz blanca y ultravioleta. Su distribución abarca algunos departamentos de las Cordilleras de los Andes (Vallejo \& Wolff 2013).

Phyllophaga pachypyga (Burmeister, 1855): Se recolectaron 2545 individuos en cuatro (RCM, CSL, RB y CUA) de los cinco fragmentos, durante abril, mayo y junio con trampas de luz blanca y ultravioleta. Su distribución es poco conocida en Colombia y sólo se habían revisado ejemplares de "Fusagasugá" sin datos adicionales (Morón 2001).

Sericini: Se caracterizan por tener el labro membranoso, transversal, casi oculto bajo el clípeo, del cual en ocasiones sólo se observa una parte del borde anterior; borde exterior de la mandíbula poco notable (Morón et al. 1997)

Astaena sp.: Es muy abundante en los fragmentos RCM y RB, donde se recolectaron 2203 individuos con trampas de luz blanca y ultravioleta.

Macrodactylini: Los adultos presentan el labro esclerosado, con el borde anterior delgado, recto o sinuado, que en su mayor parte queda cubierto por el borde anterior del clípeo; mandíbulas bien desarrolladas, con los bordes exteriores engrosados (Morón et al. 1997).

Barybas sp.: Se recolectaron 24 individuos en los fragmentos RCM, CSL y RB con ayuda de trampas de luz blanca y ultravioleta.

Liogenys quadridens (Fabricius, 1798): Es muy común por ser reconocido como plaga agrícola en el Caribe colombiano. Se recolectaron 618 individuos en RCM, CSL y DMI Luriza. Su distribución en Colombia está restringida al Caribe colombiano.

RUTELINAE

Anomalini: Presentan el labro horizontal con respecto al clípeo, y claramente separado de éste por una sutura; las antenas están constituidas por nueve antenómeros; el margen exterior de los élitros presenta un borde membranoso (Ohaus 1934, Morón et al. 1997)

Paranomala inconstans (Burmeister, 1844): Se recolectaron 1480 individuos en RCM y DMI Luriza, con ayuda de trampas de luz blanca y ultravioleta. La especie es considerada importante plaga agrícola en la zona.

Paranomala Casey, 1915: Representado por cuatro morfoespecies recolectadas en abril y mayo: Paranomala sp1 (167 individuos) se recolectó en los cinco fragmentos; Paranomala sp2 (ocho individuos), sólo en tres de ellos
(RCM, DMI Luriza y RB); Paranomala sp3 (47 individuos), se recolectó en RCM y CSL; Paranomala sp4 (93 individuos), se presentó únicamente en RCM y DMI Luriza. Las recolectas se hicieron con trampas de luz blanca y ultravioleta.

Geniatini: Son Rutelinae cuyos adultos se caracterizan por presentar el labro en posición vertical con respecto al clípeo; el labro y el mentón con una proyección media anterior ancha, cuyos ápices están en contacto mientras las piezas bucales están en reposo. Los artejos tarsales están deprimidos y ensanchados, con cojinetes ventrales de sedas cortas (Morón et al. 1997)

Leucothyreus Mac Leay, 1819: Se capturaron cuatro morfoespecies del género: Leucothyreus sp1 (siete individuos), presente en RCM, CSL y CUA; Leucothyreus sp2 (52 individuos), fue recolectada en RCM, CSL y $\mathrm{RB}$; Leucothyreus sp3 (nueve individuos), en RCM, RB y CUA; y Leucothyreus sp. 4 (un individuos), sólo en RCM.

Rutelini: Labro colocado en posición horizontal, casi paralela con respecto al clípeo, y normalmente separado de este por una sutura; antena formada por diez artejos, rara vez por ocho o nueve y el margen externo de los élitros sin borde membranoso (Morón et al. 1997, Ohaus 1934)

Pelidnota polita Latreille, 1811: Se recolectaron cinco individuos presentes en RCM y CSL durante mayo capturados con trampas de luz blanca y ultravioleta.

Xenopelidnota anomala (Burmeister, 1844): Es una especie común en el departamento durante abril y mayo. Se recolectaron 37 individuos en RCM, CSL, RB y CUA con trampas de luz blanca y ultravioleta. Se reporta por primera vez con un dato preciso para Colombia.

\section{DISCUSIÓN}

Son pocos los inventarios locales de estos escarabajos realizados en Colombia. Los resultados obtenidos en el presente estudio incrementan los registros sobre la diversidad genérica del departamento del Atlántico, ya que en los inventarios realizados por Restrepo-Giraldo et al. (2003), Suárez \& Amat (2007) y Gasca \& Amat (2010), sólo se citaron cuatro géneros de Rutelinae (Bolax, Callistethus, Paranomala y Pelidnota), tres de Dynastinae (Tomarus, Ancognatha y Cyclocephala), uno de Cetoniinae (Cotinis) y ninguno para la subfamilia Melolonthinae.

Las especies de Melolonthinae encontradas en el presente estudio, se caracterizan por ser abundantes en el departamento del Atlántico y ser reconocidas entre los campesinos y agricultores como importantes plagas de cultivos tradicionales. Sin embargo, la degradación del hábitat que trae consigo la homogeneización del mismo, pone en riesgo las poblaciones de especies con hábitos de 
alimentación y refugios restringidos, que podrían desaparecer con la extensión de las actividades antrópicas en la zona de estudio.

El nuevo reporte de $T$. cuniculus constituye un aporte importante para el conocimiento de los Pentodontini en el país, ya que Escalona \& Joly (2006) reportaba una distribución para centro y Suramérica, lo que daba una sospecha que podría encontrarse en Colombia. Sin embargo, es necesario ampliar los muestreos en el área de su recolecta para confirmar el registro, aunque los caracteres son evidentes en el individuo revisado. Con respecto a $X$. anomala, Soula (2009) presenta su descripción pero en la etiqueta sólo se refería a "Colombia" como localidad, por lo que se confirma que los fragmentos de bosque seco del departamento del Atlántico constituyen el hábitat de la especie y lo más probable es que su distribución abarque el Caribe colombiano. Falta información de su Biología, lo que podría considerarse en futuras investigaciones. Algo similar sucede con $P$. pachypyga cuyo holotipo procede de "Fusagasugá” (Morón 2001) municipio de la cordillera oriental, por lo que encontrarla en ecosistemas con menos de 200 msnm con altas abundancias es un aporte importante al conocimiento de su distribución.

Es necesario que, para ampliar el número de especies del presente inventario, se intensifiquen los muestreos y el número de trampas para la recolecta de escarabajos frugívoros de la Subfamilia Cetoniinae. A pesar de eso, el número de especies capturadas (32), correspondería al 64\% registradas para la región Caribe y 6.6\% de todo el territorio colombiano. Teniendo en cuenta lo anterior, el departamento del Atlántico alberga un número representativo de especies y géneros de escarabajos fitófagos (Scarabaeidae), por lo que se hace necesario el mantenimiento de estas zonas como refugio y hábitat para las especies de la familia y así contribuir a la conservación de la biodiversidad del Caribe colombiano.

Agradecimientos. Los autores agradecen al Departamento administrativo de Ciencia y Tecnología COLCIENCIAS que por medio de su programa Jóvenes Investigadores Virginia Gutiérrez de Pineda 2012 contribuyó a la realización de este trabajo. Además al apoyo financiero y logístico de la Vicerrectoría de Investigaciones, Extensión y Proyección social de la Universidad del Atlántico. Al Semillero de Investigaciones NEOPTERA del Caribe colombiano por todo su apoyo, especialmente a Edgar Ortíz Ruiz, Jorge Rangel, María Cristina Román, Luis Arteaga Blanco y Ana Cristina De la Parra por su acompañamiento en campo. A los habitantes de los corregimientos visitados por facilitar la estancia en esos lugares durante la realización del trabajo de campo. A Margarita López García por sus aportes al documento y ayuda a la identificación de algunas especies, a Alexander Miranda por la traducción cuidadosa del resumen y a los árbitros anónimos por dedicar su tiempo a la revisión del manuscrito, por sus importantes correcciones y recomendaciones.

\section{LITERATURA CITADA}

Endrödi, S. 1966. Monographie der Dynastinae (Col. Lam) 1 Teil. Ent. Abh. Mus. Tierk. Dresden, Bd. 33: 1-457.

Endrödi, S. 1985. The Dynastinae of the World. Dr W Junk Publishers. Dordrecht, Boston, Lancaster. 800pp.

Escalona, H.E. \& Joly, L. J. 2006. El género Ligyrus Burmeister, 1847 en Venezuela (Coleoptera: Scarabaeidae: Dynastinae:Pentodontini). Boletín Sociedad Entomológica Aragonesa, 39: 111137.

Gasca-Álvarez, H. J. \& Amat-García, G. 2010. Synopsis and key to the genera of dynastinae (Coleoptera, Scarabaeoidea, Scarabaeidae) of Colombia. In: Ratcliffe, B. \& Krell, F-T. (Eds.). Current advances in Scarabaeoidea research. ZooKeys, 34: 153-192.

Morón, M. A. 1985. Los insectos degradadores, un factor poco estudiado en los bosques de México. Folia Entomológica Mexicana, 65: 131-137.

Morón, M. A. 1994. Fauna de Coleoptera Lamellicornia en las montañas del noreste de Hidalgo, México. Acta Zoológica Mexicana (n. s.) 63: 7-59.

Morón, M. A. 2001. Revision of the rugipennis group of Phyllophaga (sensu stricto) (Coleoptera: Melolonthidae). Annals of the Entomological Society of America, 94: 771-808.

Morón, M. A., Ratcliffe, B. \& Deloya, C. 1997. Atlas de los escarabajos de México: Coleoptera Lamellicornia, Volumen I: Familia Melolonthidae. Sociedad Mexicana de Entomología, A.C. Xalapa, Veracruz, México: 280pp.

Ohaus, F. 1934. Genera Insectorum, Coleoptera, fam. Scarabaeidae: subfam. Rutelinae. Erster Teil, fasc. 199A. 172pp. 6pls.

Orozco, J. 2012. Monographic revision of the American genus Euphoria Burmeister 1842 (Coleoptera: Scarabaeidae: Cetoniinae). The Coleopterists Boulletin, (11): 1-182.

Pardo-Locarno, L. C. 2003. Escarabajos (Coleoptera: Melolonthidae) del plan aluvial del Río Cauca, Colombia I. Ensamblaje, fichas bioecológicas, extinciones locales y claves para adultos. Dugesiana, 20: 1-15.

Pardo-Locarno, L. C., Montoya, J. \& Schoonhoven, A. 2003a. Comparación de la abundancia de chisas rizófagas (Coleoptera: Melolonthidae) en agroecosistemas de Caldono, Cauca, Colombia. In: Aragón, G. A., M.A. Morón y A. Marín J. (Eds.). Estudios sobre Coleópteros del suelo en América. Publicación especial de la Benemérita Universidad Autónoma de Puebla, México.

Pardo-Locarno, L. C., Morón, M. A., Gaigl, A. \& Belloti, A. 2003b. Los complejos regionales de Melolonthidae (Coleoptera) Rizófagos en Colombia. In: Aragón, G. A., M.A. Morón y A. Marín J. (Eds.). Estudios sobre Coleópteros del suelo en América. 2003. Publicación especial de la Benemérita Universidad Autónoma de Puebla, México, 45-63.

Pardo-Locarno, L. C., Montoya, J., Schoonhoven, A. \& Morón, M. A. 2003c. Composición y riqueza del complejo Melolonthidae (Coleoptera) en cuatro agro-ecosistemas del Cauca, Colombia. In: Aragón, G. A., M.A. Morón y A. Marín J. (Eds.). Estudios sobre Coleópteros del suelo en América. 2003. Publicación especial de la Benemérita Universidad Autónoma de Puebla, México, 29-43.

Pardo-Locarno, L. C., Morón, M. A. \& Montoya-Lerma, J. 2007. Descripción de los estados inmaduros de Astaena valida (Coleoptera: Melolonthidae: Melolonthinae: Sericini). Acta Zoológica Mexicana (n.s), 23: 129-141.

Pardo-Locarno, L. C., González, J. C., Pérez, C. R., Yepes, F. \& Fernández, C. 2012. Escarabajos de importancia agrícola (Coleoptera: Melolonthidae) en la región Caribe colombiana: Regis- 
tros y propuestas de manejo. Boletín del Museo Entomológico Francisco Luis Gallejo, 4: 7-23.

Ramírez-Ponce, A. \& Morón, M. A. 2009. Relaciones filogenéticas del género Anomala (Coleoptera: Melolonthidae: Rutelinae). Revista Mexicana de Biodiversidad, 80: 357-394.

Restrepo- Giraldo, H., Morón, M. A., Vallejo, F., Pardo-Locarno, L. C. \& López-Ávila, A. 2003. Catálogo de Coleoptera Melolonthidae (Scarabaeidae Pleurosticti) de Colombia. Folia Entomologica Mexicana, 42: 239-263.

Sanabria García, R., Gasca-Álvarez, H. J. \& Amat-García, G. 2012. Sinopsis de la Tribu Oryctini (Coleoptera: Scarabaeidae: Dynastinae) de Colombia. Insecta Mundi, 0276: 1-64.
Soula, M. 2009. Review of Pelidnotina 3. The Beetles of the New World, Rutelini 3. Saintry, AECFT. 137 pp (in French).

Suárez, M. A. \& Amat, G. 2007. Lista de especies de escarabajos fruteros (Melolonthidae: Cetoniinae) de Colombia. Biota colombiana, 8: 69-76.

Útima, O. \& Vallejo, L. F. 2008. Escarabajos Melolonthidae (Scarabaeidae- Pleurosticti) de la montaña cafetera, departamento de Risaralda, Colombia. Agronómica, 16: 31-44.

Vallejo, L.F \& Wolff, M. 2013. The genus Phyllophaga Harris (Coleoptera: Scarabaeidae: Melolonthinae) in the Colombian Andean Mountain. Zootaxa (2): 101-142.

\section{APÉNDICE I}
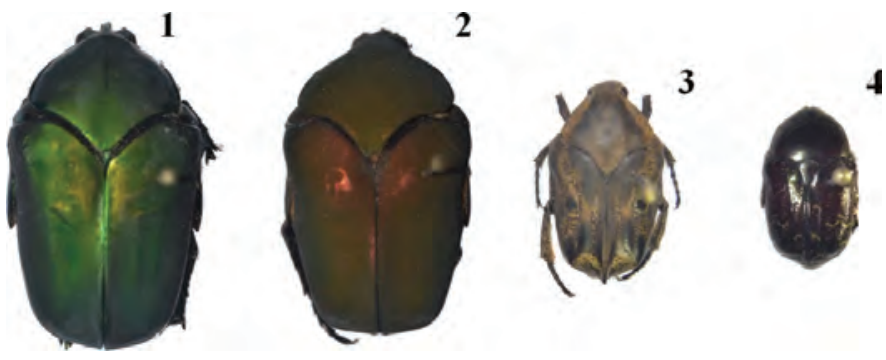

CETONIINAE. (1) Cotinis barthelemyi, (2) Cotinis lebasi, (3) Hoplopyga liturata, (4) Euphoria yucateca

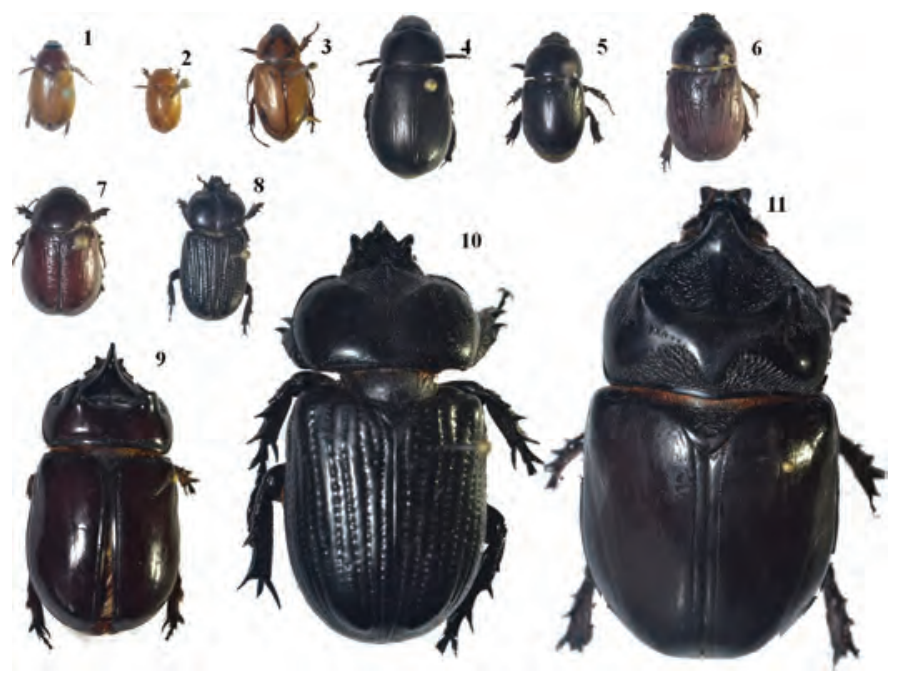

DYNASTINAE. (1) Cyclocephala melanocephala, (2) Cyclocephala ovulum, (3) Cyclocephala sp, (4) Dyscinetus dubius, (5) Euetheola humilis, (6) Tomarus cuniculus, (7) Tomarus fossor, (8) Hemiphileurus sp, (9) Strategus aloeus, (10) Phileurus didymus, (11) Strategus jugurtha.
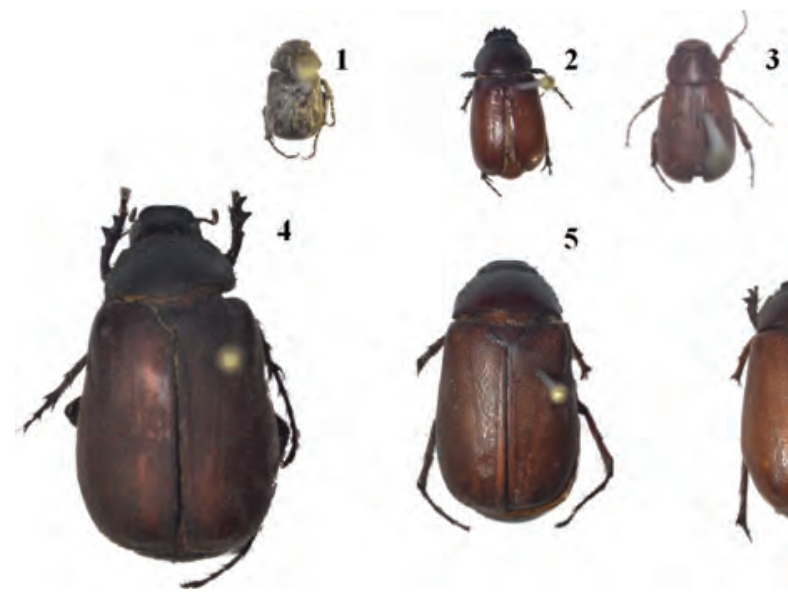

3

MELOLONTHINAE. (1) Barybas sp, (2) Liogenys quadridens, (3) Astaena sp, (4) Phyllophaga (Phyllophaga) menetriesi, (5) Phyllophaga impressipyga, (6) Phyllophaga pachypyga,

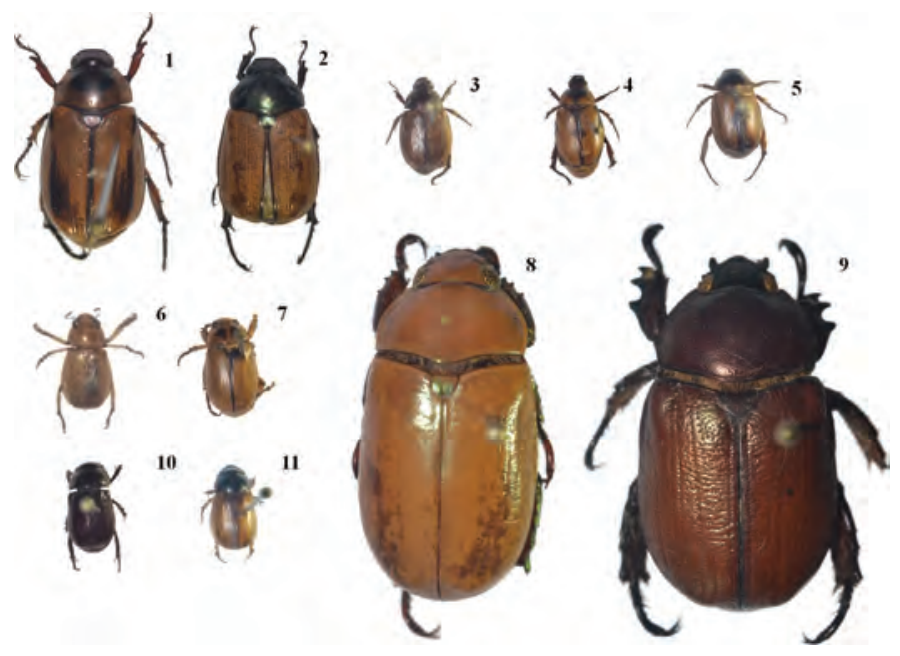

RUTELINAE. (1) Paranomala inconstans, (2) Paranomala sp1, (3) Paranomala sp2, (4) Paranomala sp3, (5) Paranomala sp4, (6) Leucothyreus sp1, (7) Leucothyreus sp2, (8) Pelidnota polita, (9) Xenopelidnota anomala (10).Leucothyreus sp3, (11) Leucothyreus sp4. 